\title{
As orientações recebidas na estratégia de saúde da família e outros serviços de saúde no âmbito da saúde da criança
}

The orientations received in family health strategies and other health services under the children's health

La orientación recibida en la estrategia de salud familiar y de otros servicios de salud en el área de la salud del niño

Liliane da Consolação Campos Ribeiro 1 ${ }^{\text {; Fabiana Antélica de Paula }}{ }^{2}$; Mariana Botelho Leite ${ }^{3}$; Bábara Ribeiro Barbosa ${ }^{4}$

\section{Resumo}

Trata-se de uma pesquisa descritiva sobre as orientações recebidas pelos responsáveis por crianças com idade entre zero a seis anos nos serviços de saúde durante os atendimentos à criança, sendo tais orientações sobre a saúde da criança, crescimento e desenvolvimento e prevenção de acidentes. O objetivo foi comparar as unidades de saúde da família com outros serviços de saúde em relação às orientações. Utilizou-se o questionário validado sobre os atributos da atenção primária à saúde denominado PCAtool. Foi verificado que os responsáveis em geral são as mães $(87,8 \%)$, com baixo nível de escolaridade (73,2\% com primário completo/ginasial incompleto) e têm como serviço de referência a unidade de saúde da família (77,6\%). Apesar do número elevado de entrevistados que têm a unidade de saúde da família como referência, ao se analisar a quantidade de orientações recebidas por serviço de referência, constatou-se que os outros serviços de saúde orientam mais os usuários. O valor não está dentro do esperado, pois a atenção básica, através da unidade de saúde da família, deveria prestar mais serviços de educação em saúde e prevenção de agravos do que os demais serviços.

Descritores: Saúde da Criança, Educação em saúde, Serviços de Saúde.

\section{Abstract}

This is a descriptive research on the guidelines received by parents of children aged between 0 and 6 years in health services during visits to the child, and such guidelines on child health, growth and development and prevention of accidents. The objective was to compare the units of family health with other health services in relation to the guidelines. We used the validated questionnaire on the attributes of primary care called PCATool. It was found that the guardians are usually the mothers

\footnotetext{
${ }^{1}$ Doutora em Ciências da Saúde (Área de Concentração: Saúde da Criança e do Adolescente) pela Universidade Federal de Minas Gerais (UFMG). Professor Adjunto do Curso de Graduação em Enfermagem - Campus JK, Diamantina, MG. Rodovia MGT 367 - KM 583, n. 5000, Alto da Jacuba, CEP: 39.100-000. Departamento de Enfermagem. email: liliane.consolcacao@ufvjm.edu.br

${ }^{2}$ Mestre em Saúde e Sociedade e Meio ambiente pela Universidade Federal dos Vales do Jequitinhonha e Mucuri (UFVJM). Professor Assistente do Curso de Graduação em Enfermagem - Campus JK, Diamantina - MG. Rodovia MGT 367- KM 583, n.5000, Alto da Jacuba, CEP: 39.100-000. Departamento de Enfermagem. email: fabiana.angelica@ufvjm.edu.br

${ }^{3}$ Enfermeira pela Universidade Federal dos Vales do Jequitinhonha e Mucuri (UFVJM). email: mariana.botelho@ufvjm.edu.br

${ }^{4}$ Graduanda de Enfermagem da Universidade Federal dos Vales do Jequitinhonha e Mucuri (UFVJM). Curso de Graduação em Enfermagem - Campus JK, Diamantina, MG. Rodovia MGT 367 - KM 583, n. 5000, Alto da Jacuba, CEP: 39.100-000. email: barbararibeiro@ufvjm.edu.br
} 
ISSN 2179-6750

(87.8\%), with low education (73.2\% with primary complete/incomplete high school) and are the reference service is the family health unit (77.6\%). Despite the high number of respondents who have a family health unit as a reference, when analyzing the amount of guidance received by the reference service, it was found that other health care guide more users. The value is not within the expected, because primary care through the family health unit should provide more services in health education and disease prevention than other services.

Key-words: Child Health, Health Education, Health Services

\section{Resumen}

Se trata de una investigación descriptiva sobre las orientaciones recibidas por los padres de los niños y niñas de cero a seis años de los servicios de salud durante las visitas al niño, y esa orientación en la salud, el crecimiento y el desarrollo del niño y prevenir accidentes. El objetivo fue comparar las unidades de salud de la familia con otros servicios de salud en relación con las directrices. Se utilizó el cuestionario validado en los atributos de la atención primaria de llamadas PCATool. Se ha encontrado que los responsables son por lo general las madres $(87,8 \%)$, bajo nivel de educación (73.2\% con primaria completa/incompleta junior) y tienen como un servicio de la familia unidad de salud de referencia $(77,6 \%)$. A pesar del alto número de encuestados que tienen la unidad de salud de la familia como una referencia en el análisis de la cantidad de orientación recibida por el servicio de referencia, se encontró que otros servicios de salud guían a más usuarios. El valor no es la esperada, debido a que la atención primaria por unidad de salud de la familia, debe prestar más servicios de educación en la prevención de la salud y la enfermedad que los otros servicios.

Palabras-claves: Salud Infantil, Educación para la Salud, Servicios de Salud

\section{Introdução}

A atenção básica à saúde tem como princípio ações individuais e coletivas baseadas em promoção e proteção à saúde, prevenção de agravos, tratamento e manutenção da saúde e tem a Estratégia de Saúde da Família (ESF) como responsável pelos serviços assistenciais ${ }^{1}$.

A relevância da promoção da saúde e prevenção de agravos deve ser considerada, visto que o tratamento e manutenção da saúde são importantes, entretanto a redução dos riscos à saúde pode diminuir a necessidade do tratamento, melhorar a qualidade de vida e a eficácia do serviço prestado 1 .

A saúde da criança constitui uma área estratégica de atuação da ESF. Trata-se de uma faixa etária com maior sensibilidade aos agravos, englobando fatores internos e externos ${ }^{2}$. Por isso, constitui um grupo que merece atenção dedicada e qualificada, envolvendo vários profissionais de saúde, dentre eles o enfermeiro.

Fatores como a escuta qualificada, desempenho clínico dos profissionais e interação da equipe durante a assistência à saúde na ESF contribuem com o vínculo entre profissionais e 
ISSN 2179-6750

cuidadores de crianças menores de cinco anos. Porém, problemas como a inadequação do espaço físico das unidades de saúde dificultam os atendimentos, prejudicando o vínculo e, consequentemente, as orientações prestadas durante trabalhos de promoção da saúde ${ }^{3}$.

É preciso que o profissional não só desenvolva o cuidado, mas seja criativo para conseguir a continuidade do acompanhamento, mantendo a singularidade do binômio criança-mãe e da família 4

A atenção à saúde da criança deve envolver a vigilância do desenvolvimento e orientação sobre possíveis desvios dos padrões de normalidade, o que possibilita detecção e intervenção precoce $^{5}$.

Além do crescimento/desenvolvimento, orientações em relação aos agravos e causas externas também são de suma importância, visto que, as crianças quando acometidas são mais sensíveis, o que dificulta a recuperação da saúde e em alguns casos pode levar ao óbito ${ }^{2}$.

Dentre os agravos e causas externas que atingem essa faixa etária, os acidentes domésticos merecem destaque. Apesar de ter indicadores elevados, o acidente doméstico ainda é interpretado como obra do destino, acaso, ou é tratado como algo comum para faixa etária ${ }^{2,6}$.

É importante destacar que se trata de um sério problema de saúde pública, pois os acidentes são muito frequentes e as taxas de morbimortalidade são elevadas. A enfermagem tem um papel importante na prevenção dos acidentes, sendo possível aproveitar vários momentos para realizar a educação em saúde, seja no cuidado domiciliar, palestras para público específico, consultas de enfermagem e, não menos importante, nos atendimentos e tratamentos hospitalares ${ }^{2}$.

As orientações quanto à saúde da criança podem ser prestadas antes mesmo do nascimento do bebê, como nos grupos de gestantes. Muitas vezes o atendimento pré-natal é voltado para a assistência curativa, quando deveria ser pautado também na promoção e prevenção ${ }^{7}$.

Entretanto, apenas orientar o responsável pela criança não é suficiente. A maneira como o profissional conduz a consulta também deve ser considerada. É preciso que as informações passadas sejam compreendidas pela família e contribuam de alguma maneira para a saúde da criança. Para tal, deve-se utilizar de linguagem clara considerando o nível social e a escolaridade da família, a fim de que as orientações recebidas sejam aplicadas no cotidiano.

O profissional deve utilizar de estratégias para verificar a compreensão do cuidador em relação às informações prestadas, uma maneira simples de avaliar esse entendimento, consiste em, ao final do atendimento, indagar sobre o que foi orientado ao longo da consulta ${ }^{3}$. Porém, o método ainda é pouco utilizado, talvez porque o profissional não considere importante ou não tenha conhecimento de tal estratégia. 
ISSN 2179-6750

Em relação à educação em saúde, é importante ressaltar que não é objetivo da ESF voltar-se apenas para a consulta individualizada, mas utilizar-se também de grupos e atividades lúdicas que sensibilizem a família e a criança ${ }^{6}$.

Tendo em vista os aspectos descritos acima, é possível ressaltar que a atenção à saúde da criança não deve se restringir apenas às ações de acompanhamento do crescimento da criança, tratase de uma fase da vida que merece atenção envolvendo os demais integrantes da família. As orientações prestadas e, principalmente, a compreensão do que é informado pelo profissional de saúde devem ser abordadas com ênfase na prevenção de agravos e na identificação precoce de problemas no desenvolvimento. Além disso, todos os momentos de contato com a equipe de saúde devem ser proveitosos no sentido de orientar, seja na atenção primária ou nos atendimentos ambulatoriais ou hospitalares.

O objetivo deste estudo foi identificar se os responsáveis pelas crianças de zero a seis anos recebem orientações sobre como manter a criança saudável, como prevenir acidentes dentro e fora do domicílio e como lidar com o crescimento e desenvolvimento das crianças. Além disso, o estudo compara as orientações prestadas em equipes de saúde da família com aquelas prestadas em outros serviços de saúde.

\section{Métodos}

Trata-se de uma pesquisa do tipo transversal realizada nas ESF do município de Diamantina, Minas Gerais, localizada na região do Alto Jequitinhonha.

A população pesquisada foi composta por responsáveis por crianças e cadastradas nas cinco Equipes de Saúde da Família totalizando 384 crianças, sendo utilizado o seguinte critério de inclusão: crianças entre zero a seis anos de idade, uma criança por domicílio, sendo que, quando houve mais de uma criança/domicílio incluiu-se a mais velha. Para selecionar as crianças utilizou-se amostragem aleatória sistemática estratificada.

O instrumento utilizado para coleta de dados realizada entre maio e junho de 2009 foi elaborado e validado no Brasil por Harzheim (2004) e denomina-se Instrumento de Avaliação da Atenção Primária (PCATool). Trata-se de um questionário com perguntas semi-estruturadas e de fácil compreensão que se aplica ao cuidador principal da criança em seu domicílio.

Aos entrevistados foram feitas perguntas sobre as orientações que já foram recebidas durante os atendimentos à criança, são elas: A) Orientações sobre como mandar a criança saudável; B) Orientações sobre manter a criança segura de tombos e afastada do fogão; C) Orientações sobre segurança doméstica, como guardar remédios com segurança; D) Maneiras de lidar com o 
ISSN 2179-6750

comportamento da criança; E) Orientações sobre mudanças no crescimento e desenvolvimento da criança.

O entrevistado teve a opção de escolher entre cinco respostas, sendo elas: com certeza sim, acho que sim, acho que não, com certeza não, não sei/não me lembro. Este estudo avaliou a frequência de respostas 'com certeza sim' para cada tipo de orientação dentre as citadas anteriormente.

De acordo com o objetivo proposto, as informações coletadas foram digitadas e analisadas em um banco de dados no software SPSS versão 18.

O projeto foi aprovado pelo comitê de ética em pesquisa da Universidade Federal de Minas Gerais (UFMG) sob o ${ }^{\circ}$ ETIC 643/2008.

\section{Resultados}

A pesquisa avaliou se os responsáveis pelas crianças recebem orientações a respeito da saúde das mesmas nas ESF e nos outros serviços de saúde.

Em relação à amostra pesquisada de 384 crianças, 77,6\% dos entrevistados ( $\mathrm{n}=298)$ afirmaram utilizar a unidade de saúde da família como serviço de referência para o atendimento da criança. Os 22,4\% restantes ( $\mathrm{n}=86)$ utilizam outros serviços de referência.

Quanto ao cuidador, o qual se considerou a pessoa mais responsável pela saúde da criança, $87,8 \%$ destes são mães, seguidos das avós (7,6\%).

A escolaridade dos cuidadores é de 73,2\% com primário completo/ginasial incompleto, seguido de $12,2 \%$ com colegial completo/superior incompleto, sendo que apenas 2,4\% possuem superior completo.

Quanto às orientações que com certeza foram recebidas, a que obteve mais respostas 'com certeza sim' foi sobre como manter a criança saudável $(79,9 \%)$ e a orientação que menos foi recebida durante os atendimentos foi sobre as maneiras de lidar com o comportamento da criança $(41,4 \%)$ (Tabela 1$)$.

Ao analisar apenas as respostas 'com certeza sim' por tipo de serviço, pode-se verificar que, dentre aqueles que têm como referência a unidade de saúde da família (n=298), 79,5\% foram orientados sobre como manter a criança saudável, enquanto apenas 39,9\% receberam orientações sobre como lidar com o comportamento da criança (Tabela 2).

Dentre os entrevistados que têm como referência outros serviços ( $n=86), 81,4 \%$ foram orientados sobre como manter a criança saudável, enquanto apenas 46,5\% receberam orientações sobre como lidar com o comportamento da criança (Tabela 2). 
Tabela 1. Análise da frequência de respostas em relação às orientações prestadas durante o atendimento à criança. Diamantina, Minas Gerais, 2009.

\begin{tabular}{|cccccccc}
\hline Orientações Recebidas sobre: & \multicolumn{2}{c}{ Com certeza sim } & \multicolumn{2}{c}{ Outras respostas } & \multicolumn{2}{c}{ Total } \\
\hline & $\mathbf{N}$ & $\mathbf{\%}$ & $\mathbf{n}$ & $\mathbf{\%}$ & $\mathbf{n}$ & $\mathbf{\%}$ \\
\hline Manter criança saudável & 307 & 79,9 & 77 & 20,1 & 384 & 100 \\
\hline $\begin{array}{c}\text { Manter criança segura de } \\
\text { tombos, afastada do fogão }\end{array}$ & 221 & 57,6 & 163 & 42,4 & 384 & 100 \\
$\begin{array}{c}\text { Guardar remédios com } \\
\text { segurança }\end{array}$ & 208 & 54,2 & 176 & 45,8 & 384 & 100 \\
\hline $\begin{array}{c}\text { Comportamento da criança } \\
\text { Mudanças no crescimento e } \\
\text { desenvolvimento da criança }\end{array}$ & 159 & 41,4 & 225 & 58,6 & 384 & 100 \\
\hline & Fonte: dados da pesquisa & & & & & \\
\hline
\end{tabular}

Tabela 2. Comparação entre a orientações recebidas pelo cuidador na Unidade de Saúde da Família e em Outros Serviços. Diamantina, Minas Gerais, 2009.

\begin{tabular}{|ccccc}
\hline Tipo de orientação recebida & \multicolumn{3}{c}{$\begin{array}{c}\text { Unidade de Saúde da } \\
\text { Família }\end{array}$} & \multicolumn{2}{c}{$\begin{array}{c}\text { Outros Serviços de } \\
\text { saúde }\end{array}$} \\
\cline { 2 - 5 } & $\mathbf{n}$ & $\mathbf{\%}$ & $\mathbf{n}$ & $\%$ \\
\hline manter criança saudável & 237 & 79,5 & 70 & 81,4 \\
\hline $\begin{array}{c}\text { manter criança segura de } \\
\text { tombos, afastada do fogão }\end{array}$ & 169 & 56,7 & 52 & 60,5 \\
\hline $\begin{array}{c}\text { guardar remédios com } \\
\text { segurança }\end{array}$ & 166 & 55,7 & 42 & 48,8 \\
\hline $\begin{array}{c}\text { comportamento da criança } \\
\text { Mudanças no crescimento e }\end{array}$ & 119 & 39,9 & 40 & 46,5 \\
desenvolvimento da criança & 159 & 53,4 & 46 & 53,5 \\
\hline
\end{tabular}

Fonte: dados da pesquisa

\section{Discussão}

O serviço de referência, que consiste no serviço preferencial de escolha para o atendimento à saúde da criança, em grande parte é a unidade de saúde da família. Esse resultado também foi identificado em outro estudo ${ }^{8}$. Assim, é possível afirmar que a acessibilidade aos serviços de atenção básica está de acordo com o esperado, mas os valores poderiam ser maiores, visto que o objetivo da saúde da família é o contato preferencial dos usuários com os serviços de saúde ${ }^{1}$.

O nível de escolaridade dos cuidadores de crianças influencia fortemente na saúde das mesmas ${ }^{9,10}$. Neste estudo, observou-se que o nível de escolaridade dos entrevistados é baixo, podendo estar relacionado às orientações recebidas durante os atendimentos, visto que, provavelmente, nem sempre o que é orientado é compreendido pelos cuidadores. 
ISSN 2179-6750

A consulta de enfermagem à criança é um momento importante para realização da educação em saúde. Outro estudo ${ }^{11}$ constatou que em $81,2 \%$ das consultas de enfermagem as mães foram orientadas sobre o crescimento e desenvolvimento da criança. Sendo assim, os resultados aqui encontrados não foram satisfatórios, visto que apenas 53,5\% dos entrevistados afirmaram receber orientações sobre o assunto.

Orientações sobre manter a criança saudável obtiveram valor mais elevado quando comparadas às demais. Apesar de representar o maior valor encontrado o resultado ainda não é positivo, pois orientações sobre aspectos essenciais à saúde da criança deveriam contemplar todos os atendimentos, a fim de aprimorá-los ${ }^{11}$.

As orientações menos recebidas foram em relação ao comportamento da criança. $\mathrm{O}$ resultado é preocupante, pois os problemas comportamentais na infância estão diretamente relacionados à escolaridade dos pais ${ }^{12}$. Portanto, sendo a população pesquisada de baixa escolaridade, o assunto deveria ser tratado com maior atenção durante os atendimentos.

As duas variáveis sobre a manter a criança segura foram tratadas de maneira semelhante nos atendimentos. Os valores de orientações que com certeza foram recebidas sobre o assunto estiveram bem próximos $(57,6 \%$ e 54,2\%). Apesar de estar acima dos 50\%, o valor poderia ser maior, pois as orientações sobre prevenção de acidentes estão interligadas àquelas sobre crescimento, desenvolvimento e necessidades das crianças. A falta dessas orientações favorece a ocorrência de acidentes domésticos ${ }^{13,14,15}$.

Um estudo em um pronto socorro pediátrico concluiu que $10 \%$ dos atendimentos prestados tinham como causa os acidentes. O valor é significativo, pois envolve uma causa evitável por meio da prevenção. Constatou-se também que crianças do sexo masculino estão mais passíveis e que grande parte dos acidentes envolve quedas, além de acidentes com corpo estranho, queimadura, intoxicação medicamentosa, atropelamento, entre outros ${ }^{16}$.

Em relação ao serviço de referência, tanto na unidade de saúde da família quanto em outros serviços, as orientações mais recebidas foram sobre como manter a criança saudável $(79,5 \%$ e $81,4 \%$, respectivamente). As menos recebidas foram sobre maneiras de lidar com o comportamento da criança (39,9\% na unidade de saúde da família e 48,8\% em outros serviços). Ainda foi possível perceber através dos resultados que, com exceção das orientações sobre segurança doméstica, todas as outras foram recebidas em menor quantidade na unidade de saúde da família.

A partir desse resultado foi possível verificar que a unidade de saúde da família não está cumprindo adequadamente com seu papel no que diz respeito à educação e promoção da saúde e prevenção de agravos ${ }^{1}$. Esperava-se que a unidade de saúde da família apresentasse resultados 
ISSN 2179-6750

maiores do que os outros serviços quanto às orientações. Isso não exclui o papel dos demais serviços no trabalho de promoção da saúde e prevenção de agravos. Entretanto, sendo a atenção básica o contato preferencial dos usuários, o ideal é que a educação em saúde seja trabalhada com maior ênfase nas unidades de saúde da família do que em outros serviços.

Em relação às orientações recebidas nos outros serviços de saúde o resultado foi bastante favorável, pois, a educação em saúde ainda é pouco utilizada em serviços de saúde que não estão ligados à atenção básica ${ }^{16}$. Neste estudo observou-se resultado contrário; os outros serviços utilizaram da educação em saúde mais do que as unidades de saúde da família.

\section{Conclusão}

Esse estudo identificou que a saúde da criança, como parte da política nacional de atenção básica, deve ser tratada com atenção especial em relação ao crescimento e desenvolvimento das mesmas e prevenção de acidentes e agravos à saúde.

Uma das formas de se trabalhar a promoção à saúde da criança consiste nas orientações prestadas durante os atendimentos, seja nas unidades de saúde da família, seja em outros serviços.

Com o objetivo de obter e analisar dados sobre orientações recebidas durante os atendimentos, esse estudo encontrou resultados sobre tais orientações e a proporção em que as mesmas são trabalhadas nos serviços de saúde, em especial nas unidades de saúde da família.

Os resultados demonstram que a maioria dos entrevistados são mães, com grau de escolaridade pouco elevado e que têm como serviço de referência a unidade de saúde da família.

As orientações sobre manter a criança saudável obtiveram valores mais satisfatórios, ainda não estando adequados, pois esse tipo de orientação deveria ser passada durante todos os atendimentos.

Outros tipos de orientações sobre comportamento da criança, crescimento e desenvolvimento e prevenção de acidentes domésticos obtiveram valores menores, ultrapassando pouco mais de $50 \%$. Isso implica em maior ocorrência de problemas de saúde que poderiam ser evitados

Quando se compara a quantidade de orientações recebidas nas unidades de saúde da família com outros serviços, conclui-se que os outros serviços de saúde estão orientando mais os seus usuários do que as unidades de saúde da família. Tal resultado é desfavorável, pois a atenção básica, que presta serviço por meio da saúde da família, tem maior responsabilidade de orientar os usuários dentre todos os serviços de saúde.

Portanto, é preciso que as unidades de saúde da família melhorem os serviços de educação 43 
ISSN 2179-6750

em saúde, orientando mais os seus usuários quanto à saúde da criança, evitando, assim, agravos e acidentes nessa fase da vida e, consequentemente, prejuízos futuros.

\section{Referências}

1. Ministério da Saúde (Brasil), Secretaria de Atenção Básica, Política Nacional de Atenção Básica. Brasília: Ministério da Saúde, 2012.

2. Pereira SFA, Carcia CA. Prevenção de acidentes domésticos na infância. Rev Enferm UNISA 2009; 10(2): 172-7.

3. Pina JC, Mello DF, Mishima SM, Lunardelo SR. Contribuições da estratégia Atenção Integrada às Doenças Prevalentes na Infância ao acolhimento de crianças menores de cinco anos. Acta Paul Enferm 2009; 22(2):142-148.

4. Mello DF, Lima RAG, Scochi CGS. Health follow-up of children in poverty situation: between the routine and eventuality of daily care. Rev Latino-am Enfermagem 2007; 15(número especial):820-827.

5. Ribeiro AM, Silva RRF, Puccini RF. Conhecimentos e práticas de profissionais sobre desenvolvimento da criança na Atenção Básica à Saúde. Rev Paul Pediatr 2010; 28(2):208214.

6. Acker JIBV, Cartana MHF. Construção da participação comunitária para a prevenção de acidentes domésticos infantis. Rev Bras Enferm 2009; 62(1):64-70.

7. Melo JM, Brandão EHS, Dutra SMV, Iwazawa AT, Albuquerque RS. Conhecendo a captação de informações de mães sobre cuidados com o bebê na estratégia Saúde da Família. Texto Contexto Enferm 2007; 16(2):280-286.

8. Harhein E. Evaluación de la atención a la salud infantil del Programa Saúde da Familia en la región sur de Porto Alegre [Tese de Doutorado]. Espanha: Departamento de Salud Pública, Universidade de Alicante; 2004, 189f.

9. Monteiro CA, Benício MHD, Konno SC, Silva ACF, Lima ALL, Conde WL. Causas do declínio da desnutrição infantil no Brasil, 1996-2007. Rev Saúde Pública 2009; 43(1):35-43.

10. Damião JJ. Influência da escolaridade e do trabalho maternos no aleitamento materno exclusivo. Rev Bras Epidemiol 2008: 11(3):442-452.

11. Saparolli ECL, Adami NP. Avaliação da qualidade da consulta de enfermagem à criança no Programa de Saúde da Família. Acta Paul Enferm 2007; 20(1):55-61.

12. Assis SG, Avanci JQ, Oliveira RVC. Desigualdades socioeconômicas e saúde mental infantil. Rev Saúde Pública 2009;43(Supl. 1):92-100.

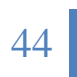


ISSN 2179-6750

13. Souza LJEX, Barroso MGT. Envenenar é mais perigoso: uma abordagem etnográfica. Cogitare Enferm 1998; 3(1):13-20.

14. Souza LJEX, Rodrigues AKC, Barroso MGT. A família vivenciando o acidente doméstico: relato de uma experiência. Rev Latino-am enferm 2000; 8(1):83-89.

15. Fonseca SS, Victora CG, Halpern R, Barros AJD, Lima RC, Monteiro LA, Barros F. Fatores de risco para injúrias acidentais em pré-escolares. J Pediatr 2002; 78(2):97-104.

16. Filócomo FRF, Harada MJCS, Silva CV, Pedreira MLG. Estudo dos acidentes na infância em um pronto socorro pediátrico. Rev Latino-am Enfermagem 2002; 10(1):41-47. 